\title{
Diagnóstico de la comunicación interna y la participación en sistemas de gestión de la calidad en dos entidades del sector público colombiano*
}

\section{Internal communication and participation in quality management systems diagnosis in two Colombian public organizations}

Adriana del Pilar Casas Henao**

Procuraduría General de la Nación

José Jorge Roca Martíne******

Superintendencia de Puertos y Transporte

\section{RESUMEN}

Este documento aborda el diagnóstico de la gestión de la comunicación interna frente a su papel para promover que los servidores participen en la implementación de los sistemas de gestión de la calidad en dos entidades públicas que usaron la Norma Técnica de Calidad y Gestión Pública (NTCGP) 1000:2009. Se presentan los resultados del diagnóstico elaborado para determinar el papel de la comunicación interna como parte

* Artículo resultado de revisión.

** Comunicadora Social y Periodista; especialista en Gerencia de Procesos y Calidad y magíster (c) en Calidad y Gestión Integral. Profesional especializada de la Procuraduría General de la Nación. Correo electrónico: nanitacasashenao@gmail.com.

***Administrador Público; especialista en Instituciones Jurídico Políticas y Derecho Público y magíster (c) en Calidad y Gestión Integral. Jefe Oficina de Control Interno, de la Superintendencia de Puertos y Transporte. Correo electrónico: jjrocamart@gmail.com 
esencial del proceso de implementación de los sistemas de gestión en las dos entidades objeto de estudio y su incidencia en la participación de los servidores públicos, tomando como punto de partida el análisis de las practicas, los contenidos y desarrollos de la comunicación en dichas instituciones; esto para determinar su integración y alineación con las estrategias, la política y los objetivos de calidad. Para ello la comunicación interna se analiza desde sus dimensiones estratégica, operativa, cultural y de aprendizaje, y considerando factores como la comprensión, el compromiso, la toma de conciencia, el sentido de pertenencia e incidencia. Como resultado se presentan las conclusiones sobre la descripción de las dinámicas de la comunicación interna encontradas en las entidades estudiadas y las de la medición de los aspectos asociados a la participación de los servidores públicos en la implantación de sus sistemas de gestión de calidad y además, los instrumentos de investigación, como aporte a la comprensión de la realidad comunicativa y su relación con la cultura de la calidad, así como bases para la presentación de alternativas metodológicas que faciliten el desarrollo de los sistemas de gestión de la calidad mediante la participación de los servidores públicos en el sector gubernamental colombiano.

Palabras clave: gestión de la calidad, sistemas de gestión de calidad, comunicación interna, participación, gestión pública.

\section{ABSTRACT}

This document addresses the diagnosis of the internal communication management versus its role to promote the participation of the servers in the implementation of the quality management systems of two public entities that implemented this management tool under the Norma Técnica de Calidad NTCGP 1000:
2009, which was created to comply with what was established in Law 872 of 2003, regarding the implementation of a Quality Management System applicable to the executive branch of public power and other Entities providing services, in the Colombian government sector. This article presents the results derived from the diagnosis developed to determine the role of internal communication as an essential part of the implementation process of the management systems in the two entities under study and their incidence in the participation of public servants in the Taking as its starting point the analysis of the practices, contents and developments of the communication in these two institutions, to determine their integration and alignment with the strategies and with the policy and quality objectives. For this purpose, internal communication was analyzed from its strategic, operative, cultural and learning dimensions and considering factors such as understanding, commitment, awareness, sense of belonging and incidence which, according to the theoretical referents adopted, constitute determinants to facilitate the participation of the employees within its organization and in its SGC. As a result of this research, we present the main conclusions about the description of internal communication dynamics found in the studied entities, the conclusions of the measurement aspects associated with the participation of public servants in the implementation of their systems Of quality management and in addition, the research instruments are presented as a contribution to the understanding of the communicative reality and its relation with the culture of quality, as well as bases for the presentation of methodological alternatives that facilitate the development of the systems of Quality management through the participation of public servants in the Colombian government sector.

Keywords: Quality management, internal communication, participation, public management. 


\section{INTRODUCCIÓN}

A partir de la expedición de la Constitución Políitica de 1991 las entidades del sector público colombiano inician un proceso de transformación de sus modelos de gestión y gerencia. Al respecto, el artículo 209 establece que "la función administrativa está al servicio de los intereses generales y se desarrolla con fundamento en los principios de igualdad, moralidad, eficacia, economía, celeridad, imparcialidad y publicidad, mediante la descentralización, la delegación y la desconcentración de funciones" (Aguilera, 2014, s. p.). Al igual que el sector estatal latinoamericano, Colombia tomó como referente las experiencias de países como Japón, Estados Unidos, Inglaterra y España e incorporó iniciativas y herramientas como la adopción de modelos de calidad total, reingeniería y sistemas de desarrollo administrativo, para mejorar la gestión de las entidades estatales.

De esta forma, la administración pública de Colombia inició un proceso de modernización en el que se articuló la calidad como eje para el desarrollo de una gestión ágil, transparente y de cara al ciudadano. También se iniciaron procesos de transformación relacionados con la gestión de la información y del talento humano.

Desde los años noventa Colombia dio inicio al desarrollo de instrumentos legales como el Sistema de Desarrollo Administrativo (Sisteda), adelantado con la expedición de la Ley 498 de 1998, la cual estableció normas sobre la organización y el funcionamiento de las entidades de orden nacional; la adopción del Modelo Estándar de Control Interno (MECI), adoptado mediante el Decreto 1599 de 2005 (actualizado en 2014), en el cual se establecen los conceptos, metodologías y herramientas que proporcionan la estructura básica para evaluar la estrategia, la gestión y los mecanismos de evaluación de la gestión pública, y la adopción de los sistema de gestión de calidad a partir de la Ley 872 de 2003 y la Norma Técnica de
Calidad en la Gestión Pública NTCGP 1000:2004 y su segunda versión en 2009, con fundamento en criterios de mejoramiento continuo, enfoque por procesos, participación ciudadana y comunicación organizacional. Esta normatividad se constituye en el eje que regula la implementación de la calidad como modelo de gerencia pública y determina los niveles de participación y colectividad a través de la comunicación, en las entidades objeto de este estudio.

De manera particular, la NTCGP 1000:2009 y la nueva versión del MECI (2014) precisan el papel de la comunicación interna como parte esencial dentro de una organización y destacan el compromiso de la alta dirección en esta materia, en aras de garantizar la difusión de las políticas institucionales y de la información relevante dentro de la organización a través de canales que resulten eficaces para estos fines.

Estas iniciativas de modernización de la gestión pública colombiana encuentran sus fuentes en los procesos de calidad iniciados en Europa en los años noventa, como la Carta de Servicios en Inglaterra y los departamentos de calidad en España (Rodríguez, 2012). En América Latina sobresale la Carta Iberoamericana de Calidad en la Gestión Pública, que trae entre sus objetivos "promover un enfoque común en Iberoamérica sobre la calidad y la excelencia en la gestión pública" (Centro Latinoamericano de Administración para el Desarrollo [CLAD], 2008), desde el cual fundamentó la adopción de estos modelos de gestión en el marco de la denominada nueva gestión pública, y la creación de los premios nacionales de calidad en países como Argentina, Brasil, Chile, Colombia, Costa Rica, Ecuador, entre otros.

Uno de los elementos recurrentes en estos modelos de gestión implementados en Colombia es la comunicación interna, considerada como esencial para promover la participación en la gestión y la democratización de la 
información pública (Sisteda), que tiene una dimensión estratégica al vincular la entidad con su entorno y facilitar la ejecución de sus operaciones internas (Congreso de Colombia, 2014) y para la cual se deben establecer procesos considerando la eficacia, la eficiencia y la efectividad del sistema de gestión de la calidad (NTCGP 1000:2009).

Una revisión al estado del arte sobre este tema permite ubicar casi de manera única y exclusiva dos estudios sobre la implementación de los sistemas de gestión de calidad ( $\mathrm{SGC}$ ) y su relación con la comunicación interna, el primero adelantado por la firma Image People en el Distrito Capital (Aguilera y Orjuela, 2008) cuyos resultados mostraron que un $80 \%$ de las entidades presentaban problemas de gestión relacionados con la comunicación; el segundo, realizado por Casals \& Asociados Inc., como base para el Modelo de Comunicación Pública Organizacional e Informativa para Entidades del Estado (MCPOI) (Usaid - Casals \& Associates Inc., 2004), en el que se identificaron como barreras de la comunicación organizacional su desarticulación con la cultura organizacional y los problemas en el manejo de la información, y su interacción comunicativa con la sociedad a través de los medios.

En concordancia con lo anterior, la Contraloría General de la República, al evaluar el nivel y la implementación y consolidación de los SGC y del MECI en diferentes entidades estatales, valoró el papel de la comunicación interna y advirtió en su informe para la vigencia 2013 debilidades de los sistemas de control de las entidades públicas auditadas, relacionadas con deficiencias en la comunicación interna entre dependencias. Estas traen fallas en el debido y oportuno flujo de información, en la documentación que deben reportar las áreas y problemas en la comunicación entre las áreas misionales y las de apoyo; todo ello genera procesos incompletos o inoportunos en el quehacer institucional (Contraloría General de la República, 2015).
Aunque se observa que desde el estado del arte y los estudios gubernamentales son pocas las investigaciones realizadas frente a la articulación de la calidad, comunicación y participación en el sector gubernamental colombiano, de esta revisión se concluye que si bien se ha indagado por las limitaciones en los flujos de comunicaciones en las entidades del sector público colombiano, aún no se han examinado con mayor detenimiento las características de la comunicación interna asociada a los sistemas de gestión de la calidad, como tampoco los aspectos asociados a la participación de los servidores públicos en estos sistemas, como resultado de los procesos de comunicación interna.

Teniendo en cuenta lo anterior, se tomaron como referentes de investigación dos entidades de la administración pública colombiana (la primera, un órgano de control de carácter autónomo y la segunda, perteneciente a la rama ejecutiva del orden nacional, según la estructura del estado colombiano) para analizar el papel que cumple la comunicación interna en el marco de los SGC de estas dos organizaciones y, a su vez, determinar cómo esta contribuye en la promoción de la participación del personal en dichos sistemas.

En este ámbito, se explora otro aspecto relacionado con la implementación del SGC en el sector público, como la comunicación, lo cual aporta al estudio de la gerencia al servicio del Estado, de las instituciones públicas y de la ciudadanía en general, y plantea una línea de partida para medir la funcionalidad, el uso y el impacto de la comunicación interna como factor determinante en la implementación de un SGC.

Considerando además que la comunicación interna es un elemento fundamental en la toma de decisiones y en la gestión del cambio, la investigación involucra otras relevancias de carácter teórico, metodológico, práctico y social. Desde el punto de vista teórico, su importancia se deriva de la oportunidad de aplicar la 
teoría de la complejidad en los sistemas planteada por Morín (1994), donde la comunicación interna se presenta como un elemento hologramático, recursivo y dialógico de toda organización. Además, se plantea un enfoque participativo (Jackson y Welch, 2007) y estratégico de la comunicación interna (Pérez, 2012).

En el ámbito metodológico, la investigación brinda una serie de instrumentos y herramientas para medir la comunicación interna y valorar su contribución con el desarrollo de un SGC en el sector público. Sus implicaciones prácticas se relacionan con su contribución a la implementación de la NTCGP 1000:2009, pero también con la ambientación para adoptar la norma ISO 9001:2015 en el sector público, de tal forma que se evidencien los beneficios de un SGC y que este no sea visto simplemente como una obligación.

Desde estas perspectivas, la investigación tiene como objetivo principal presentar el diagnóstico de la comunicación interna y su relevancia en promover la participación de los servidores en la implementación de los SGC en dos entidades objeto de estudio, describiendo sus dinámicas de comunicación interna, valorando los aspectos más importantes asociados a esta participación y adelantando un ejercicio práctico que permita sentar las bases de futuras propuesta metodológicas de implementación de sistemas de gestión de la calidad, desde fundamentos de participación y comunicación organizacional.

\section{METODOLOGÍA}

\section{Elementos teóricos y conceptuales}

El desarrollo de la investigación se fundamentó en el paradigma histórico hermenéutico (Bernal Torres, 2000), que se aparta de lo experimental y se concentra en entender e interpretar los fenómenos y las realidades sociales a partir del significado que sus protagonistas les otorgan a estos acontecimientos (Bernal Torres, 2010). El enfoque de la investigación es mixto de diseño no experimental transaccional descriptivo, ya que la medición es de carácter cuantitativo y cualitativo, y son empleados de forma simultánea (Hernández, Fernández y Baptista, 2014); esto con el propósito de obtener información de la realidad estudiada desde diferentes perspectivas y conseguir datos de diversa índole que complementan el análisis.

Desde lo teórico y lo conceptual, la investigación se desarrolla desde tres ámbitos: el primero asociado a la evolución del concepto de organizaciones, entendidas como sistemas sociales complejos; el segundo, referido a la comunicación interna como proceso de carácter estratégico capaz de permear la cultura organizacional, y el tercero, que aborda la participación del personal entendida como el objetivo general de la comunicación interna y elemento esencial para implementar y desarrollar un SGC.

\section{Ámbito de las organizaciones como sistemas complejos}

Abordar la comunicación en las organizaciones implica entender sus procesos básicos de gestión, sus dinámicas internas y externas, especificidades y su papel frente al entorno. Estas son concebidas como sistemas complejos, que según el investigador de complejidad Paul Cilliers (1998) se caracterizan por la suma de un conjunto de elementos interdependientes que interactúan de forma múltiple, producen e intercambian información, se retroalimentan y operan en lo que él ha denominado una estabilidad dinámica.

Dado su carácter abierto, las organizaciones tanto públicas como privadas, fluyen de acuerdo con las dinámicas de su entorno y, en esa medida, como lo señala Morín, son impactadas por dicho entorno, permeadas 
por la incertidumbre permanente generada por su contexto y sus propias dinámicas (De Almeida, 2008).

Las características de la organización, entendida como un sistema abierto y complejo impactado por el cambio, implican abordar la comunicación como un proceso de carácter estratégico en las instituciones que cumple una función articuladora y que, desde el ámbito de su competencia, coadyuva al cumplimiento de la misión y los objetivos de cualquier organización.

Como lo advierte Morín "Hablamos de la comunicación y es evidente que tiene que ver con la organización y la organización con la acción. No se concibe la estrategia como organización de la acción sin la comunicación. Son partes inseparables" (Pérez, 2008, p. 1).

Lo anterior implica entender la comunicación como un proceso inherente a cualquier organización que, con el paso del tiempo y el desarrollo de las tecnologías de la información, ha adquirido un carácter estratégico fundamental en la toma de decisiones y en la gestión del cambio organizacional. Así, la comunicación, y específicamente la comunicación interna, se constituye en un proceso que construye las organizaciones, las mantiene y les permite evolucionar.

Entender las entidades públicas como sistemas complejos también implica definir los fundamentos que deben orientar la comunicación interna para que esta sea concebida como un elemento sustancial de la organización. Para ello, se consideraron los principios del pensamiento complejo propuestos por Morín (citado por Elorriaga, Lugo y Montero, 2012): la hologramática, en la medida que la comunicación interna es inherente a cualquier organización, fluye a través y fuera de ella, y al mismo tiempo la define y la particulariza; la dialógica, pues la comunicación interna debe considerar toda la información de carácter organizacional y permitir su constatación, contrastación y validación, y la recursividad, si se considera que la comunicación interna se caracteriza por ser dinámica y en esa medida suministra información que da respuesta a múltiples interrogantes, que a su vez genera más preguntas e inquietudes y, por ende, nuevas incertidumbres

\section{Ámbito de la comunicación interna en las organizaciones}

El lenguaje y los modos de comunicarse son los pilares básicos de la cultura de una organización (Marín, 1997) y de la construcción colectiva de su realidad. Esta última, según el enfoque interpretacionista de la comunicación, es un proceso de construcción de significados comunes que fundamenta las acciones de los miembros de las organizaciones dentro de su entorno laboral. Esa construcción colectiva de la realidad es lo que da origen a la denominada cultura organizacional.

Los sociólogos Peter Berger y Thomas Luckmann (1999) plantean desde el constructivismo social que la comunicación es realmente significativa para construir la realidad social. Por un lado, se debe tener en cuenta que la comunicación, inmersa en las relaciones personales, es la base de los consensos y al mismo tiempo, a través de la comunicación, se fijan las pautas de la convivencia y participación.

Desde estas premisas, es innegable el carácter transversal de la comunicación frente a toda la organización. Su función integradora hace de esta una herramienta necesaria para consolidar la gestión de calidad en el sector público. Como lo señala Nosnik, la comunicación debe ser entendida como un apoyo estratégico y, en este contexto, este proceso debe penetrar en todos los niveles de la organización o entidad pública, y concientizar a sus miembros acerca de la importancia de su labor en el desarrollo de la empresa. Vista así,

La comunicación estratégica es una forma de pensar sistémicamente en cualquier nivel, ya sea personal, grupal y social. Este pensamiento sistémico obliga a concebir el 
proceso de comunicación como un sistema, para lo cual se necesita estar consciente de todas las partes que la integran (Pérez, 2012, s. p.).

Lo anterior implica abordar la comunicación interna no solo desde lo estratégico sino también desde sus otras dimensiones. En ese sentido, se tomó en consideración el modelo desarrollado por el experto en comunicación interna y cultura organizacional Alejandro Formanchuk (2015) que contempla siete dimensiones de las cuales, para los propósitos del proyecto investigativo, se adaptaron cuatro: estratégica, operativa, cultural y aprendizaje.

Mirar la comunicación desde estas cuatro perspectivas implica revisar la manera como esta se articula con el plan estratégico institucional y el proyecto institucional de la entidad pública (dimensión estratégica); las prácticas de comunicación interna implementadas para movilizar al personal en torno al cumplimiento de los objetivos establecidos, los canales empleados, las características de los mensajes difundidos (dimensión operativa); los procesos de aprendizaje generados a partir de la retroalimentación a los miembros de la organización sobre su desempeño (dimensión de aprendizaje) y su papel en la generación de procesos de interacción social y la participación a distintos niveles de los integrantes de la organización (dimensión cultural), lo que se constituye en un elemento fundamental en los SGC.

\section{Ámbito de la participación como objetivo de la comunicación interna y exigencia de los sistemas de gestión de la calidad}

Jackson y Welch (2007) refuerzan la idea de que la comunicación interna tiene como propósito global la promoción de la participación de los empleados en sus instituciones. En ese sentido, la comunicación debe contribuir a las relaciones internas caracterizadas por el compromiso de los empleados. la promoción del sentido positivo de pertenencia. el desarrollo de su conciencia sobre el cambio del medio ambiente y el desarrollo de su comprensión de la necesidad de que la organización evolucione en sus objetivos (Jackson y Welch, 2007).

En consonancia con lo anterior, la NTCGP1000:2009 destaca la participación activa de los servidores públicos como una exigencia a la hora de gestionar la calidad dentro de una entidad y precisa la necesidad de asegurarse de que los integrantes de la organización pública 0 particulares que ejercen funciones públicas "[...] sean conscientes de la pertinencia e importancia de sus actividades y de cómo contribuyen al logro de los objetivos de la calidad" (Icontec, 2009).

Esta toma de conciencia se constituye en requisito esencial para propiciar dos aspectos claves a los que también hacen referencia, tanto la norma, como los objetivos de la comunicación interna planteados por Jackson y Welch (2007): el entendimiento y el compromiso de los servidores con el cambio hacia la cultura de la calidad, que se traduce en la participación activa de los servidores o particulares que ejercen funciones públicas.

En suma, la comunicación sirve de sustento y vehículo para el desarrollo de la participación en la organización, lo que lleva necesariamente a la implementación de modelos de comunicación de carácter circular que privilegien la retroalimentación. Al respecto Marín (1997) señala que el tipo de participación está mediado por el modelo de comunicación implementado. Así, "las formas participativas en las organizaciones se fundamentan en la adecuada comprensión y utilización de modelos circulares de comunicación [...]" (p 263).

En ese sentido, las organizaciones deben orientarse al desarrollo de sistemas de comunicación circulares, en 
los que las respuestas de la gente adquieren relevancia, dado que la eficacia de la comunicación depende en este caso de la perfecta comprensión del mensaje y de esto se asegura el emisor mediante el feedback, a diferencia del modelo de comunicación lineal básico en el que centra la perfecta difusión del mensaje.

Desde esta perspectiva teórica y conceptual, se analizó la comunicación interna (desde sus dimensiones estratégica, operativa, cultural y aprendizaje) de dos entes de control públicos colombianos que cuentan con SGC implementados y el rol que esta cumple en la promoción y el fortalecimiento de la participación de los servidores en sus sistemas de gestión de calidad.

\section{Momentos y desarrollo de la Investigación}

La investigación en su integridad se desarrolló a partir de cinco momentos investigativos (ver figura 1): el diseño de instrumentos de investigación, la revisión documental, el análisis de resultados, la estructuración de la propuesta de comunicación interna y la validación de la misma propuesta. En este artículo se aborda el desarrollo y los resultados de los tres primeros momentos.

Figura 1. Momentos de la investigación

Fuente: elaboración propia.

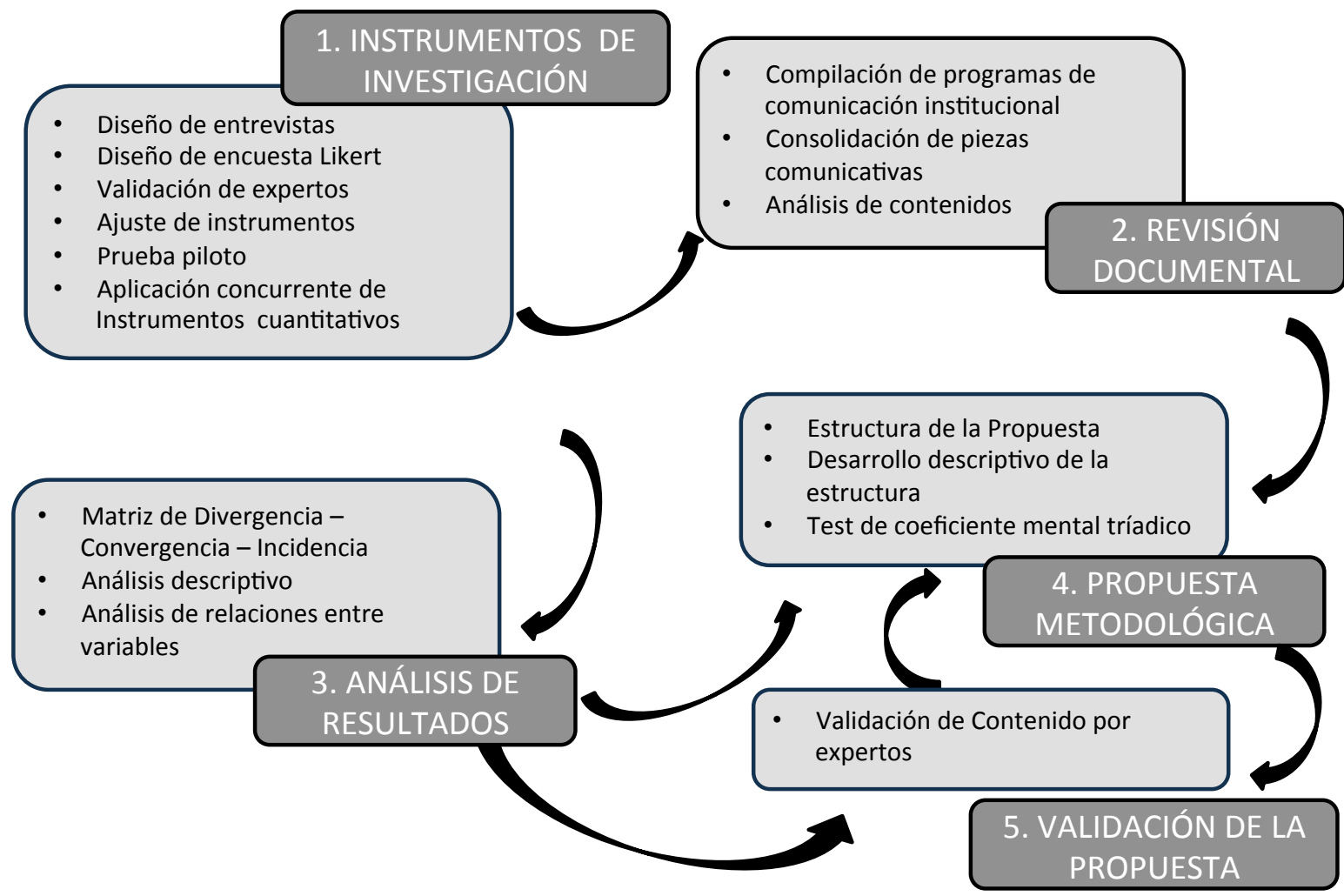


Para efectos de este estudio, se definieron y desarrollaron una serie de instrumentos (ver tabla 1) que facilitaron la descripción inicial del proceso de comunicación interna de las dos instituciones, partiendo de la revisión documental y de los resultados de la encuesta tipo Likert aplicada a los servidores de las dos entidades, la cual arrojó información acerca del tipo de medios de comunicación empleados y la percepción que poseen los funcionarios sobre la forma como se gestiona este tipo de comunicación. Se diseñaron y aplicaron además entrevistas semiestructuradas para reconocer la percepción que tenín los líderes de comunicación, control interno y gestión de la calidad en las entidades sobre la comunicación interna y su articulación con el SGC desde sus dimensiones estratégica, operativa, cultural y de aprendizaje y retroalimentación.

Entre tanto, la participación se abordó mediante una escala de Likert, que se estructuró en 25 afirmaciones distribuidas en diferentes bloques para medir la percepción de los servidores públicos sobre la comprensión del SGC, el grado de conciencia, el compromiso, sentido de pertenencia con la entidad (Jackson y Welch, 2007) y su incidencia en el destino estratégico de la organización.

Tabla 1. Instrumentos y técnicas de investigación

\begin{tabular}{|l|l|}
\hline \multicolumn{1}{|c|}{ FASES DE LA INVESTIGACIÓN } & \multicolumn{1}{|c|}{ TÉCNICAS O INSTRUMENTOS DE INVESTIGACIÓN } \\
\hline \multirow{3}{*}{ 1. Diseño y aplicación de instrumentos } & $\begin{array}{l}\text { Diseño y aplicación de tres cuestionarios para entrevistas semiestructuradas para los } \\
\text { líderes de comunicación interna, SGC y control interno, de cada entidad estudiada, } \\
\text { para medir su percepción en cuanto a comunicación y SGC. }\end{array}$ \\
\cline { 2 - 3 } 2. Revisión documental & $\begin{array}{l}\text { Diseño y aplicación de una encuesta tipo escala de Likert aplicada a la muestra } \\
\text { seleccionada de funcionarios de las entidades estudiadas, para medir su percepción } \\
\text { frente a la comunicación interna y los SGC. }\end{array}$ \\
\cline { 2 - 3 } & $\begin{array}{l}\text { Juicio de expertos: diseño de instrumentos aplicados a los instrumentos para medir sus } \\
\text { niveles de validez y confiabilidad. Se aplicó por parte de tres expertos en comunicación } \\
\text { o SGC. }\end{array}$ \\
\hline 3. Análisis de resultados & $\begin{array}{l}\text { Revisión documental (consecución y revisión de los procesos, procedimientos, } \\
\text { documentos y productos de comunicación interna y sobre la implementación y estados } \\
\text { de los SGC de cada entidad. }\end{array}$ \\
\hline $\begin{array}{l}\text { Matriz de convergencia, divergencia e incidencia: para identificar las orientaciones, } \\
\text { lineamientos y características en común o diferencias entre los procesos y acciones } \\
\text { de comunicación adoptados por las entidades de estudio, así como también aquellos } \\
\text { elementos que sin ser compartidos o implementados pueden influir de manera positiva } \\
\text { en sus SGC. }\end{array}$ \\
\hline
\end{tabular}

Fuente: elaboración propia. 
El estudio se desarrolló de forma concurrente, en la medida en que los métodos cuantitativos y cualitativos empleados se aplicaron de forma simultánea, considerando los tiempos programados para su diseño e implementación, así como las condiciones de acceso a los funcionarios de las entidades públicas.

La validez de contenido de los instrumentos de medición empleados y de la propuesta metodológica planteada (escala de Likert y tres cuestionarios de preguntas para aplicar en entrevista semiestructurada) se hizo mediante la metodología de juicio de expertos (Escobar y Cuervo, 2008) y una prueba piloto, en el caso de la escala de Likert. En el análisis de los resultados derivados de la aplicación de entrevistas se utilizó un método de triangulación de datos, bajo parámetros de comparación e inferencias cualitativas (Hernández et al., 2014) que comprende la recolección, revisión, organización, definición de unidades de análisis, codificación y descripción.

Se utilizaron tres niveles de análisis de la información para las dos entidades definidas (ver figura 2). Un primer nivel que comprendió las dimensiones de la comunicación interna (estratégica, operativa, cultural y de aprendizaje y retroalimentación); el segundo nivel referido a las perspectivas de los entrevistados (representante de la alta dirección, comunicación y control interno), y un tercer nivel que corresponde a la información resultante de la comparación de los dos primeros niveles, que fue codificada con el propósito de identificar y agrupar aquellos aspectos relevantes de la comunicación interna señalados por los entrevistados frente a cada una de las cuatro dimensiones.

Figura 2. Modelo de triangulación de datos

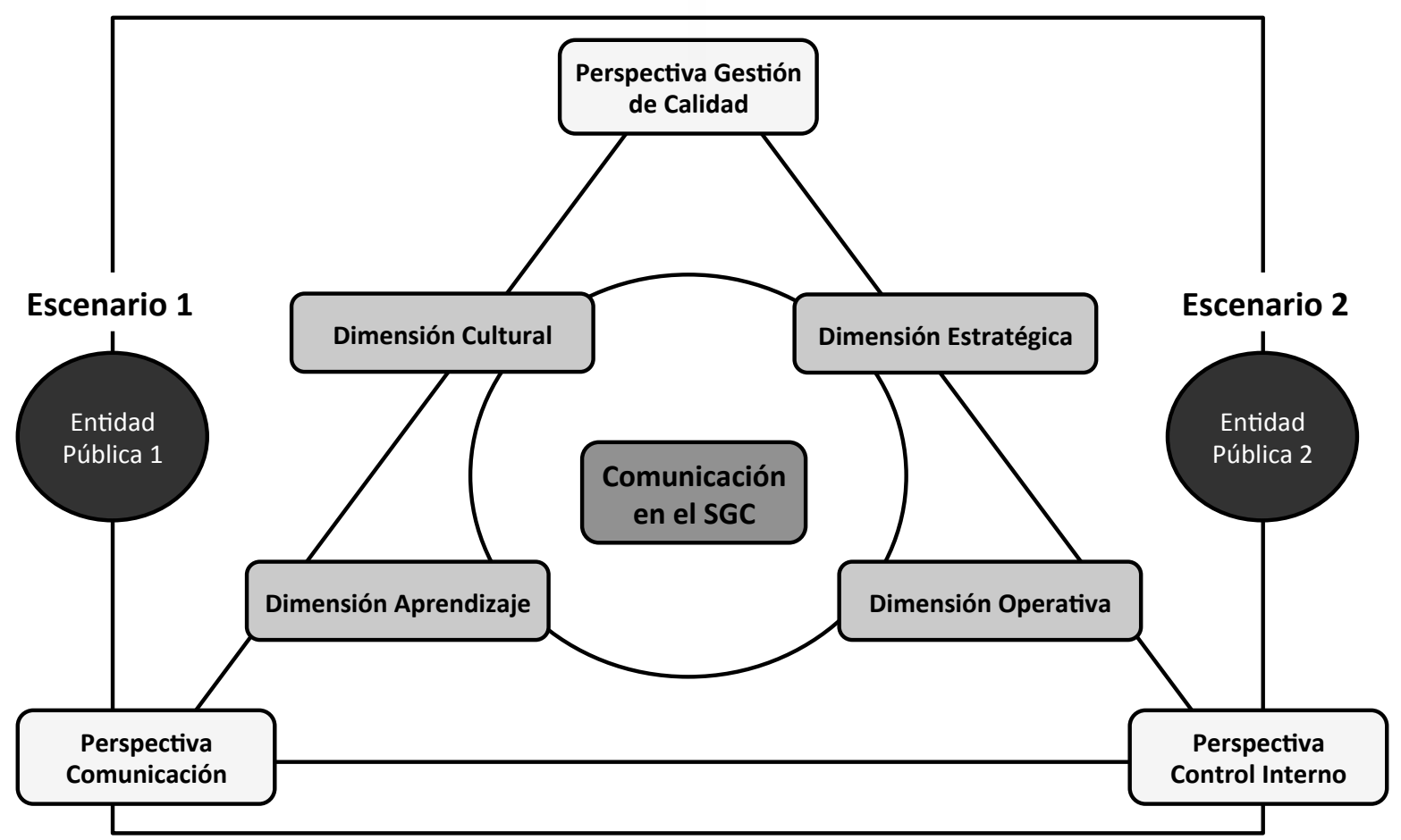

Fuente: elaboración propia. 
Los hallazgos y las conclusiones de esta investigación fundamentaron el desarrollo de una propuesta metodológica de comunicación interna planteada para las dos organizaciones.

\section{RESULTADOS Y DISCUSIÓN}

\section{Sobre el diseño de instrumentos de investigación}

Teniendo en cuenta la definición de la justificación de proyectos de investigación, se tomaron los criterios definidos por Hernández et al. (2014). En el ámbito metodológico esta investigación brinda una serie de instrumentos y herramientas para medir la comunicación interna y su contribución al desarrollo de un SGC en el sector público, la cultura del control y la participación de los servidores en la gestión de la calidad, que antes no se tenían o no eran conocidos en el ámbito de las entidades públicas en Colombia.

En efecto, la encuesta tipo escala Likert, los cuestionarios para las entrevistas semiestructuradas a los líderes de comunicación, control interno y SGC, y la matriz de triangulación de datos para determinar convergencias, divergencias e incidencias son instrumentos que las entidades públicas y privadas pueden aplicar no solo para desarrollar diagnósticos de comunicación interna, sino también para otras dimensiones de la gestión organizacional.

\section{Sobre la revisión documental}

La dinámica de la comunicación interna en cada una de las entidades públicas analizadas se abordó mediante una revisión documental sobre los desarrollos en materia de comunicación organizacional con énfasis en comunicación institucional; entrevistas semiestructuradas con los representantes de la alta dirección, líderes de comunicación organizacional y con los responsables de las oficinas de control interno, y una encuesta tipo Likert (también empleada para medir la variable de participación), que permitió conocer la percepción de los servidores públicos sobre la comunicación interna y el uso que ellos hacen de los canales de comunicación existentes en sus instituciones.

Los resultados de la revisión documental, del análisis de la información obtenida mediante las entrevistas y de la encuesta de percepción advierten una desarticulación de la comunicación interna con las políticas y objetivos estratégicos de las entidades y con las políticas y objetivos de calidad, pues resulta evidente que la comunicación se ha centrado en difundir la información sobre los resultados de la gestión y se ha dirigido fundamentalmente al público externo. No obstante, se reconoce la necesidad de alcanzar esta integración.

La revisión documental en esta materia se abordó desde cinco aspectos que comprenden los aspectos más relevantes del desarrollo de la comunicación interna que se pueden evidenciar a través de la información documentada recopilada y revisada en las dos entidades estudiadas: 1) el modelo de operación; 2) la estructura orgánica; 3) la comunicación organizacional; 4) la gestión de la comunicación, y 5) su evaluación. Se encontró que en las dos entidades existen diferencias sobre la concepción y organización del proceso de comunicación interna, tanto en los modelos de operación por procesos como en sus estructuras orgánicas. Difieren además en su formalización como elemento estratégico, pues una de ellas la concibe como un subproceso de apoyo, mientras que la otra, como parte de los procesos estratégicos. A su vez, se advierte que las políticas de comunicación interna no se han desarrollado a cabalidad en ninguna de las dos entidades. 
Se evidenció que la planeación, el seguimiento y la evaluación de la gestión de la comunicación interna son limitados y se circunscriben a verificar la ejecución de actividades y uso de canales de información. Además, es necesario que el compromiso de la alta dirección se refleje también en la asignación planificada de recursos (financieros, tecnológicos y de talento humano), de tal manera que responda a las necesidades reales de la institución, lo cual no se observó como política institucionalizada, sino más bien como práctica coyuntural en las dos entidades, pero más estructurada en la segunda de ellas.

Por otra parte, con la información derivada de las entrevistas semiestructuradas a los líderes de la gestión de la calidad, la comunicación interna y el control interno se identificaron y codificaron 24 elementos denominados atributos (ver tabla 2), los cuales son objeto de un tercer nivel de análisis; posteriormente, se establecieron las convergencias, divergencias e incidencias existentes entre estos atributos,

Tabla 2. Atributos identificados para la comunicación interna

\begin{tabular}{|l|c|l|}
\hline DIMENSIONES & CÓDIGO & \multicolumn{1}{|c|}{ ATRIBUTOS } \\
\hline \multirow{5}{*}{ Estratégica } & E1 & $\begin{array}{l}\text { Integración con la } \\
\text { estrategia de la entidad }\end{array}$ \\
\cline { 2 - 3 } & E2 & Roles y funciones \\
\cline { 2 - 3 } & E3 & $\begin{array}{l}\text { Políticas y objetivos } \\
\text { institucionales }\end{array}$ \\
\cline { 2 - 3 } & E4 & Articulación con el SGC \\
\cline { 2 - 3 } & E5 & Resultados de la gestión \\
\cline { 2 - 3 } & E6 & Compromiso institucional \\
\hline \multirow{5}{*}{ Operativa } & 01 & Gestión de la comunicación \\
\cline { 2 - 3 } & 02 & $\begin{array}{l}\text { Competencias } \\
\text { comunicativas }\end{array}$ \\
\cline { 2 - 3 } & 03 & Promoción del compromiso \\
\cline { 2 - 3 } & 04 & Información y contenidos \\
\hline
\end{tabular}

\begin{tabular}{|c|c|c|}
\hline DIMENSIONES & CÓDIGO & ATRIBUTOS \\
\hline \multirow{2}{*}{ Operativa } & 05 & $\begin{array}{l}\text { Canales y flujos de } \\
\text { información }\end{array}$ \\
\hline & O6 & $\begin{array}{l}\text { Eficacia de la } \\
\text { comunicación }\end{array}$ \\
\hline \multirow{7}{*}{ Cultural } & $\mathrm{C} 1$ & Desempeño laboral \\
\hline & $\mathrm{C}_{2}$ & Sentido de pertenencia \\
\hline & $\mathrm{C} 3$ & Cultura de la calidad \\
\hline & $\mathrm{C} 4$ & Participación \\
\hline & C5 & Promoción de la conciencia \\
\hline & C6 & Autocontrol \\
\hline & C7 & Manejo del rumor \\
\hline \multirow{5}{*}{ Aprendizaje } & $\mathrm{A} 1$ & $\begin{array}{l}\text { Resultados del } \\
\text { seguimiento }\end{array}$ \\
\hline & $\mathrm{A} 2$ & Impacto del SGC \\
\hline & A3 & Mejora del SGC \\
\hline & A4 & Retroalimentación \\
\hline & A5 & Relacionamiento \\
\hline
\end{tabular}

Fuente: elaboración propia.

\section{Convergencias, divergencias e incidencias}

Las divergencias hacen referencia a los postulados $\mathrm{u}$ opiniones discrepantes o discordantes, que se diferencian significativamente; las convergencias a las tendencias a unirse en un punto, a coincidir o aproximarse; las incidencias fueron tomadas en el sentido de la posibilidad de repercutir o causar efecto una cosa sobre otra (Real Academia de la Lengua, 2016). Como resultado del análisis de las convergencias, divergencias e incidencias se obtuvieron inferencias de carácter cualitativo sobre la gestión de la comunicación externa en las dos instituciones públicas estudiadas, las cuales se presentan a continuación. 
- En la dimensión estratégica de la comunicación interna se infiere que las dos entidades analizadas reconocen la importancia de articular la comunicación interna con las funciones, políticas y objetivos estratégicos de la entidad, pero no se ha desarrollado esta integración. Se estableció que en estas organizaciones la comunicación organizacional está orientada hacia el manejo de medios y la comunicación externa primordialmente. No obstante, se apalanca la difusión de información de los resultados de la gestión institucional derivada de los procesos misionales y de la gestión del talento humano.

En ninguna de las dos entidades se evidenció la definición de políticas y planes operativos de comunicación articulados con la estrategia institucional. En ese sentido, la comunicación interna debe integrarse a proyectos de actualización del SGC, a partir de la revisión de cadenas de valor o proyectos de rediseño organizacional y buenas prácticas, y acompañar cualquier proceso de cambio en las entidades.

- A nivel operativo se encontró que las dependencias responsables de la comunicación organizacional en estas dos instituciones dependen jerárquicamente del líder de la alta dirección. Para gestionar este proceso se emplean, en los dos casos, canales de comunicación de similares características sin diferenciación de públicos y grupos de interés, como tampoco de contenidos específicos para cada parte interesada del SGC. Se advierte además la ausencia de un número suficiente de espacios presenciales de información y consulta. No obstante, existen fortalezas en cuanto al uso de las tecnologías de la información y la comunicación (TIC) fuertemente ligado a la comunicación interna.
También se evidenció que los lineamientos y acciones de comunicación interna (manual o protocolo) no se evalúan. En ese sentido, se llama la atención sobre la necesidad de medir y hacer seguimiento a los impactos de la comunicación interna por parte de las áreas responsables. En cuanto al personal, es preciso ampliar los perfiles profesionales que se vinculan a los equipos de comunicación para darle a la comunicación interna un enfoque más interdisciplinario.

El desarrollo operativo de la comunicación interna en estas dos entidades también plantea la necesidad de asignar una partida presupuestal para el desarrollo de este proceso y hacer una mejor planificación de los recursos requeridos para su desarrollo (financieros, tecnológicos y de talento humano).

- En el cálculo de la dimensión cultural de la comunicación interna, el estudio permitió evidenciar que este tipo de comunicación no es comprendida como promotor de la cultura organizacional ni eje y elemento fundamental para promover la participación (generación de sentido de pertenencia y conciencia individual y colectiva). Se evidencian además percepciones diferentes acerca de la comunicación interna como agente dinamizador del cambio, diferencias en el manejo del chisme o el rumor dentro de la organización y se advierte la necesidad de detectar y atender los focos de distorsión informativa y las diferencias en la apropiación y comprensión de la información.

En el ámbito organizacional existe una concepción común del concepto de calidad en el colectivo como un elemento asociado con el cumplimiento de normas y requisitos. No obstante, la identificación de grupos primarios de comunicación favorece el trabajo en equipo para el SGC. 
- En cuanto a las inferencias cualitativas identificadas en la dimensión de aprendiæaje y retroalimentación de la comunicación interna, se identificaron limitaciones en los mecanismos de medición y seguimiento de la comunicación interna y pocos escenarios y canales de comunicación interna para facilitar la retroalimentación y la participación del personal. En ese sentido, en las dos entidades se identificó un predominio de flujos de información de carácter vertical descendente 0 en cascada, que responden al orden jerárquico establecido, lo que afecta la retroalimentación de la información. Además, en las organizaciones analizadas existe poca información y difusión de los resultados del SGC a través de los medios de comunicación interna existentes.

El seguimiento a la gestión de la comunicación interna se realiza a través de auditorías internas (incipientes en una organización y moderadas en la otra) y los resultados de estas presentan un bajo impacto de las acciones de mejora.

\section{Análisis sobre la percepción de los servidores sobre la comunicación interna}

De manera complementaria a la revisión documental y a la realización de las entrevistas a los líderes de los sistemas de gestión de calidad, de comunicación organizacional y de control interno, se aplicó una encuesta sobre el modelo de escala tipo Likert (que también mide aspectos asociados con la participación de los servidores en su SGG) para medir el nivel de uso de los medios de comunicación por parte de los servidores, su percepción sobre la comunicación interna y su contribución en el desarrollo e implementación del SGC (ver figura 3).

Figura 3. Percepción sobre la comunicación interna, histograma.

Entidad pública 1

\section{$52.12 \%$}

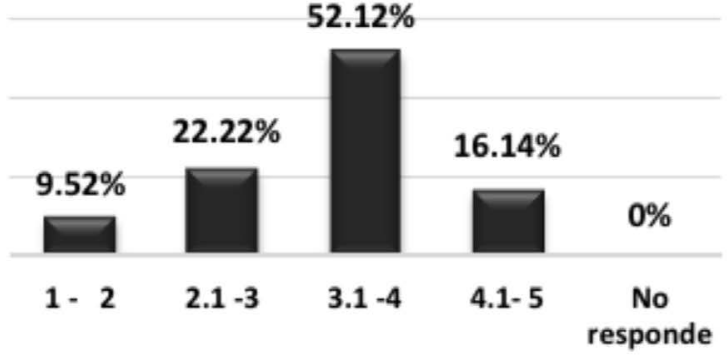

Entidad pública 2

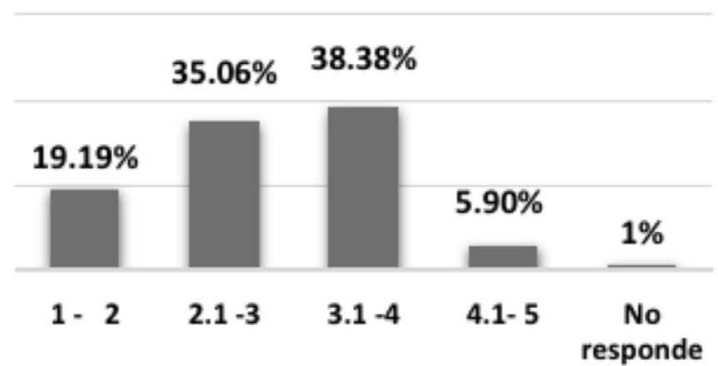

\footnotetext{
*Actitud de los encuestados en los cuatro intervalos establecidos para el análisis que oscilan entre 1 y 5 . Actitud muy desfavorable (1-2); actitud
} desfavorable (2.1-3); actitud favorable (3.1-4) y actitud muy favorable (4.1-5).

Fuente: elaboración propia. 
Los resultados de la medición de la percepción de los servidores de las dos entidades sobre la comunicación interna muestran que en la entidad 1 el 68,26\% de la población tiene una percepción positiva sobre la comunicación interna $(52,12 \%$ favorable y $16,14 \%$ muy favorable). Es decir que los contenidos resultan de interés para los destinatarios y, en términos generales, consideran que la comunicación es efectiva en su organización. No obstante, existe un $31,74 \%$ de servidores que tiene una opinión negativa sobre la forma como gestiona la comunicación interna.

Por su parte, en la entidad 2, más de la mitad de los encuestados $(54,25 \%)$ inclina negativamente la balanza con un 19,19\% de percepción muy desfavorable y un 35,06\% desfavorable. Esto demuestra el nivel de insatisfacción de los servidores con el tipo de información emitida. Se advierte además que, a juicio de los encuestados, los mensajes recibidos a través de los diferentes canales de comunicación no proporcionan información suficiente sobre el SGC y que la comunicación interna no ha contribuido a la mejora de este.

La percepción de los servidores de las dos organizaciones respecto a su comunicación interna, y específicamente a su papel como dinamizador de la información del SGC, muestra la necesidad de que los responsables de su planeación y ejecución implementen mecanismos de seguimiento, medición y evaluación de la comunicación interna y de su relación con los públicos internos. De esta manera, será posible identificar las necesidades y expectativas de los servidores, su nivel de satisfacción con los contenidos e implementar acciones de mejora orientadas a adecuar la comunicación a las necesidades de la organización.

Ahora bien, respecto de las dinámicas de los servidores frente al uso de los medios de comunicación empleados en las instituciones estudiadas, son similares. Las dos entidades gestionan su comunicación interna principalmente a través de la intranet, la página web, el correo electrónico, un sistema de carteleras, publicaciones en formato digital y reuniones de las dependencias y áreas de trabajo.

Los resultados de la aplicación de este instrumento de medición indican que el correo electrónico registra el más alto nivel de uso representado en un 95,98\% (actitudes favorables y muy favorables) para la entidad 1 y un $69,74 \%$ para la entidad 2; seguido de la página web, que en la entidad 1 registra un $85,19 \%$ de opiniones positivas y de las reuniones de grupo para la entidad 2, con un impacto favorable y muy favorable del 53,19\%. La intranet es el tercer canal de comunicación más empleado en la entidad 1 con un $81.49 \%$ (actitudes favorables y muy favorables), mientras que en la entidad 2 su uso registra uno de los niveles más bajos, con solo un $35,42 \%$ de actitudes favorables y muy favorables.

Por otra parte, las dos entidades coinciden en que los boletines virtuales y el chisme o rumor son empleados en menor medida para compartir información relacionada con acciones que afectan el entorno laboral. De hecho, el chisme o rumor tiene altos niveles de rechazo, tanto en la entidad 1 como en la entidad 2, con un $67,99 \%$ y un $74,54 \%$ de percepción negativa, respectivamente. Llama la atención que en la entidad 2 el porcentaje negativo sobre el uso de la mayoría de canales de comunicación supera las opiniones positivas sobre estos. Ejemplo de ello son las opiniones desfavorables y muy desfavorables que registran el boletín virtual $(65,32 \%)$, la intranet $(64,58 \%)$ y las carteleras institucionales $(52,03 \%)$.

Estas cifras muestran un bajo nivel de aceptación de un importante número de canales de comunicación, lo que afecta la recepción de la información emitida a través de estos y la eficacia de la comunicación interna. 
Si bien la comunicación organizacional y, por ende, la interna dependen jerárquicamente de la alta dirección, en ninguna de las dos entidades se ha concebido de manera clara una estructura de gobierno para ella. Esto se evidencia en la ausencia de planes o programas de comunicación definidos con objetivos y metas claras, la limitada planificación de recursos, la poca interdisciplinariedad de los perfiles profesionales, la poca medición de impactos, el predominio de los flujos de información de manera descendente y no transversal, y la concentración de la definición de contenidos comunicativos sin un mayor análisis de los diferentes grupos, de las partes interesadas y de sus requisitos de comunicación.

\section{Análisis sobre la participación de los servidores en los sistemas de gestión de calidad}

En concordancia con los objetivos de la investigación, se midieron los aspectos que inciden en la participación de los servidores de las dos entidades en la implementación y consolidación de su respectivo SGC. Para ello se midieron cinco dimensiones (conciencia de los empleados frente a aspectos concretos, sentido positivo de pertenencia, compromiso con el SGC, comprensión del SGC y el destino estratégico de la organización e incidencia en el desarrollo de la gestión de la organización) que propician el objetivo general de la comunicación: la participación de los empleados en sus organizaciones.

En las dos instituciones, las dimensiones asociadas con la comprensión, el sentido de pertenencia e incidencia registran resultados más bajos, pero muy similares entre sí. En el caso de la entidad 1 los resultados de estos tres aspectos oscilan entre $73 \%$ y $86 \%$, y en la entidad 2 , entre $56 \%$ y $59 \%$. Los resultados obtenidos en las dos entidades para la dimensión de comprensión (figura 4) demandan una revisión de la información emitida, de los canales de comunicación empleados y de los resultados de la implementación del SGG, para identificar las barreras que han impedido que la totalidad de los servidores comprenda la política y objetivos de calidad, así como la forma de satisfacer las necesidades del cliente y exceder sus expectativas como lo establece la NTCGP 1000:2009. La comprensión se convierte en una exigencia para garantizar una gestión eficaz; el desconocimiento del SGC por parte de los servidores limita sus posibilidades de participación.

Figura 4. Comprensión del sistema de gestión de calidad, histograma

Entidad Pública 1

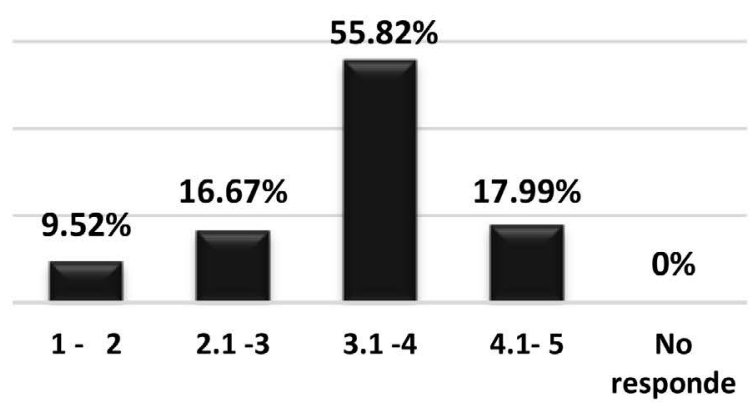

Entidad Pública 2

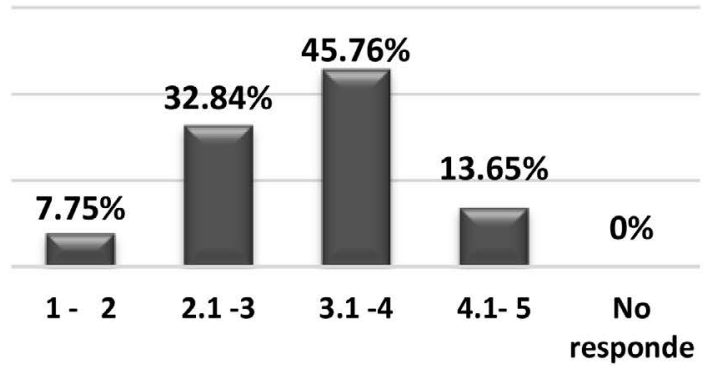

*Actitud de los encuestados en los cuatro intervalos establecidos para el análisis que oscilan entre 1 y 5 . Actitud muy desfavorable (1-2); actitud desfavorable (2.1-3); actitud favorable (3.1-4) y actitud muy favorable (4.1-5).

Fuente: elaboración propia. 
El compromiso y la toma de conciencia (figuras 5 y 6) registran los niveles más altos de favorabilidad. En el caso de la entidad 1 los resultados positivos en su conjunto corresponden al 96,83\% (compromiso) y al $92,59 \%$ (toma de conciencia) y en la entidad 2, al 86,35\% (compromiso) y al 74,17\% (toma de conciencia). El compromiso registrado por los servidores de las dos instituciones es determinante dentro de la investigación. Al respecto, cabe destacar que la NTCGP 1000:2009 señala que el compromiso se traduce en la participación activa de quienes se desempeñan en lo público.

Las entidades analizadas registran un alto nivel de conciencia acerca de los objetivos y la política de la calidad, y sobre la forma como, desde el ejercicio de sus funciones, los servidores contribuyen a su cumplimiento. No obstante, el conocimiento reflexivo sobre estos aspectos no implica necesariamente la acción y participación activa de los empleados. En este aspecto es necesario ahondar sobre los instrumentos o técnicas de participación de estas instituciones.

Se observa un grado de coherencia entre los resultados negativos obtenidos por la entidad 2 en las dimensiones de comprensión (40,59\% desfavorables y muy desfavorables), sentido de pertenencia (figura 7) $(42,06 \%$ desfavorables y muy desfavorables) e incidencia (figura 8 ) (42,43\% desfavorables y muy desfavorables). Esto indica que existe un segmento de la población que muestra una marcada inconformidad en su quehacer laboral y frente a algunos aspectos del SGC. Entre tanto, la entidad 1 registra niveles de opinión favorables en estas dos últimas dimensiones, lo que permite concluir de manera preliminar que la comunicación interna cumple con sus objetivos en esta materia.

Figura 5. Compromiso con el sistema de gestión de calidad, histograma

\section{Entidad Pública 1}

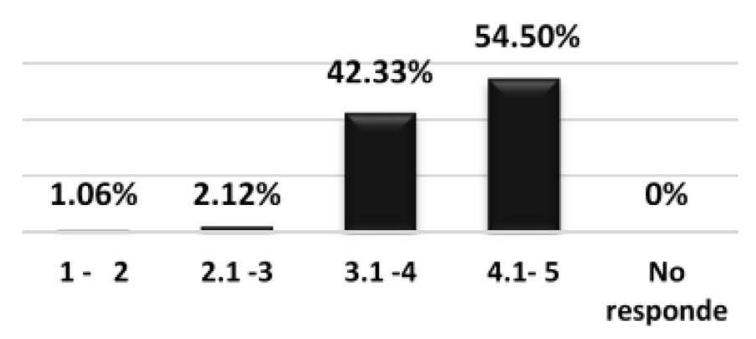

Entidad Pública 2

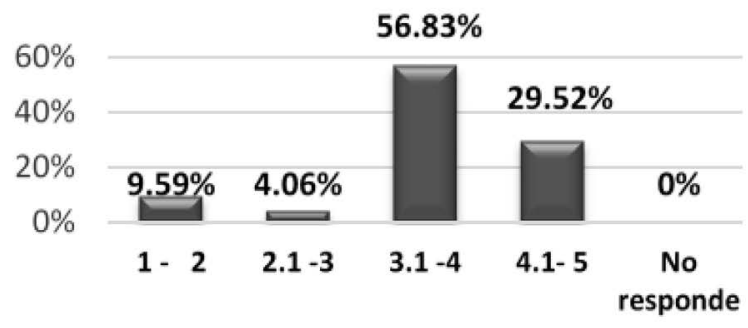

*Actitud de los encuestados en los cuatro intervalos establecidos para el análisis que oscilan entre 1 y 5 . Actitud muy desfavorable (1-2); actitud desfavorable (2.1-3); actitud favorable (3.1-4) y actitud muy favorable (4.1-5).

Fuente: elaboración propia. 
Figura 6. Conciencia sobre el sistema de gestión de calidad, histograma

\section{Entidad Pública 1}

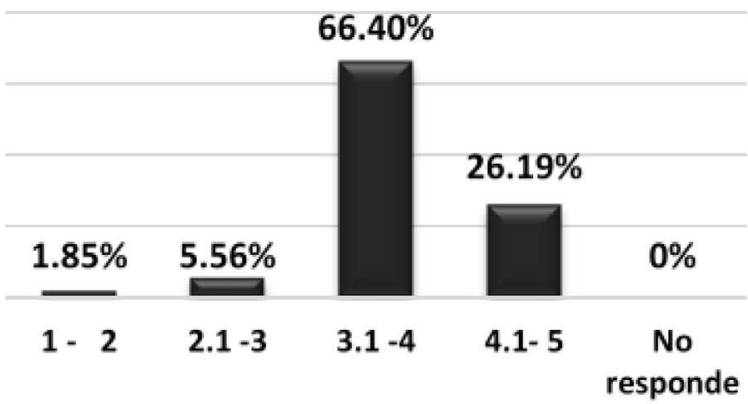

Entidad Pública 2

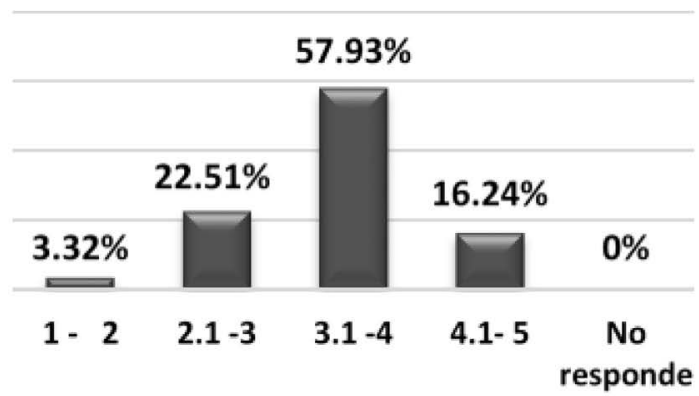

Actitud de los encuestados en los cuatro intervalos establecidos para el análisis que oscilan entre 1 y 5 . Actitud muy desfavorable (1-2); actitud desfavorable (2.1-3); actitud favorable (3.1-4) y actitud muy favorable (4.1-5).

Fuente: elaboración propia.

Figura 7. Sentido de pertenencia con el sistema de gestión de calidad, histograma

\section{Entidad Pública 1}

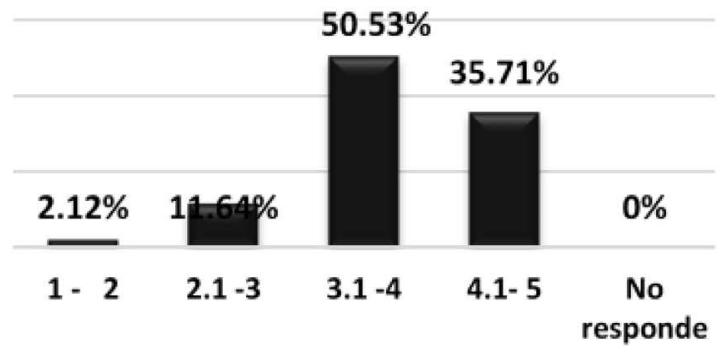

\section{Entidad Pública 2}

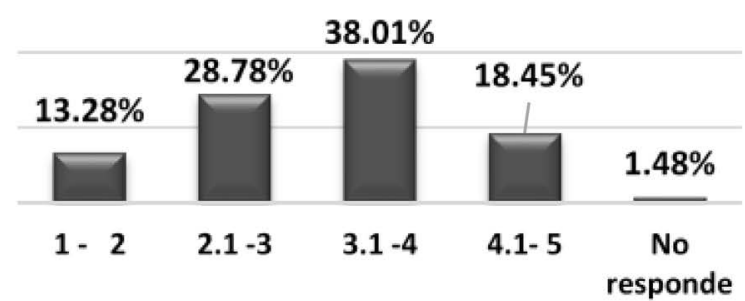

Actitud de los encuestados en los cuatro intervalos establecidos para el análisis que oscilan entre 1 y 5 . Actitud muy desfavorable (1-2); actitud desfavorable (2.1-3); actitud favorable (3.1-4) y actitud muy favorable (4.1-5).

Fuente: elaboración propia. 
Los resultados de la medición de la comunicación interna y de los aspectos asociados a la participación de los servidores en sus organizaciones orientaron la construcción de una propuesta metodológica de comunicación interna —objetivo principal de este estudio-, para promover la participación de los servidores en la implementación de los SGC en las entidades objeto de estudio.

La propuesta, validada mediante juicio de expertos, pretende alinear la comunicación interna de las organizaciones estudiadas con los respectivos componentes estratégicos institucionales, para mejorar así los flujos de información sobre la gestión de la calidad, fortalecer la participación de los servidores públicos en el diseño y desarrollo de los sistemas de gestión de calidad de sus respectivas entidades y además, proporcionar herramientas que permitan adecuar los contenidos y canales de comunicación interna requeridos por el SGC a la cultura organizacional de estas dos instituciones.

Figura 8. Incidencia en el sistema de gestión de calidad, histograma

\section{Entidad Pública 1}

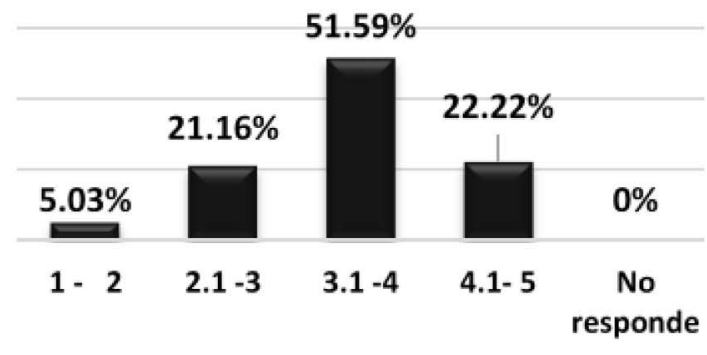

Entidad Pública 2

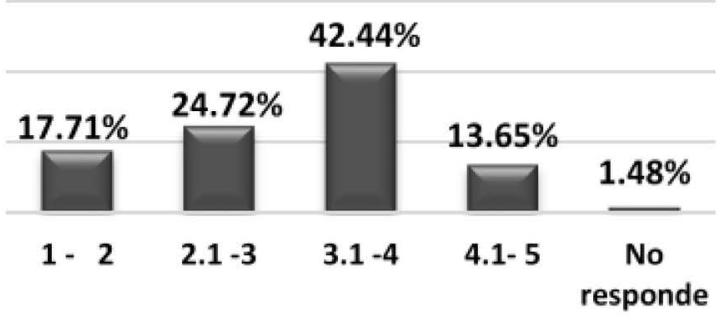

Actitud de los encuestados en los cuatro intervalos establecidos para el análisis que oscilan entre 1 y 5 . Actitud muy desfavorable (1-2); actitud desfavorable (2.1-3); actitud favorable (3.1-4) y actitud muy favorable (4.1-5).

Fuente: elaboración propia. 


\section{CONCLUSIONES}

Los resultados de la medición de la comunicación interna y de la percepción de la participación en la construcción de los sistemas de gestión de la entidades objeto de estudio evidencian una relación directa entre los objetivos específicos de la comunicación interna señalados por Jackson y Welch (2008) — compromiso, toma de conciencia, sentido de pertenencia y comprensión-, sumados a la incidencia, con las dimensiones planteadas por Formanchuk (2015) y adoptadas para esta investigación (estratégica, operativa, cultural y de aprendizaje y retroalimentación). Esto se advierte aun cuando las dos entidades tienen características muy particulares que marcan claras diferencias y que van desde su naturaleza jurídica y funciones misionales, su tamaño en presupuesto o personal. Pero en cuanto a los modelos de comunicación y participación en la implementación de sus sistemas de calidad, se observaron conductas y practicas muy similares, las cuales se señalan a continuación.

- En las dos instituciones, las dimensiones asociadas con la comprensión, el sentido de pertenencia e incidencia registran resultados más bajos, pero muy similares entre sí. En el caso de la primera entidad, los resultados de estos tres aspectos oscilan entre $73 \%$ y $86 \%$ y en la segunda, entre $56 \%$ y $59 \%$.

- Los resultados obtenidos en las dos entidades para la dimensión de comprensión demandan una revisión de la información emitida, de los canales de comunicación empleados y de los resultados de la implementación del SGC, con el propósito de identificar las barreras que han impedido que la totalidad de los servidores comprenda la política y objetivos de calidad, así como la forma de satisfacer las necesidades del cliente y exceder sus expectativas como lo establece la NTCGP 1000:2009. La comprensión se convierte en una exigencia para garantizar una gestión eficaz; el desconocimiento del SGC por parte de los servidores limita sus posibilidades de participación.

- En las dos organizaciones el compromiso y la toma de conciencia registran los niveles más altos de favorabilidad. En el caso de la primera entidad, los resultados positivos en su conjunto corresponden al 96,83\% (compromiso) y al 92,59\% (toma de conciencia), y en la segunda, al 86,35\% (compromiso) y al $74,17 \%$ (toma de conciencia). El compromiso registrado por los servidores de las dos instituciones es determinante dentro de la investigación. Al respecto, cabe destacar que la NTCGP 1000:2009 señala que el compromiso se traduce en la participación activa de quienes se desempeñan en lo público.

- Las entidades analizadas registran un alto nivel de conciencia acerca de los objetivos y la política de la calidad y sobre la forma como, desde el ejercicio de sus funciones, los servidores contribuyen a su cumplimiento. No obstante, el conocimiento reflexivo sobre estos aspectos no implica necesariamente la acción y participación activa de los empleados. En este aspecto es necesario identificar si dentro de estas instituciones existen instrumentos o técnicas de participación.

- Se observa un grado de coherencia entre los resultados negativos obtenidos por la segunda entidad en las dimensiones de comprensión (40,59\% de opiniones desfavorables y muy desfavorables), sentido de pertenencia $42,06 \%$ de opiniones desfavorables y muy desfavorables) e incidencia $(42,43 \%$ de opiniones desfavorables y muy desfavorables). Esto indica que existe un segmento de la población que muestra una inconformidad asociada a su quehacer laboral y frente a algunos aspectos del SGC que deben ser analizados por la 
alta dirección y los responsables de la comunicación interna. Entre tanto, para la primera entidad se registran niveles de opinión favorables en estas dos últimas dimensiones, lo que permite concluir de manera preliminar que la comunicación interna cumple con sus objetivos de promover un sentido positivo de pertenencia y generar espacios de comunicación donde los servidores puedan exponer sus ideas y participar e incidir en algunas decisiones que impactan la gestión de la organización.

Pese a que las dos entidades objeto de estudio han implementado modelos de gestión, se evidencia que la comunicación interna no cumple sus objetivos de manera contundente, pues se advierte una desarticulación con los elementos estratégicos de la calidad y de las propias entidades, y su dinámica no facilita el entendimiento del entorno organizacional. Además, no es considerada como un elemento estratégico al momento de evaluar los resultados de los sistemas de gestión o de control establecidos.

La información obtenida mediante las entrevistas y las encuestas permite concluir que la comunicación interna ha venido cumpliendo con una función más de tipo informativo y de consulta, que de promoción de la participación en la construcción de los sistemas de gestión de calidad.

La revisión documental evidenció que la poca información sobre los resultados e impactos de los SGC se centra en aspectos formales básicos, como la caracterización de procesos y procedimientos, usos de formatos y registros. En este sentido, la comunicación interna no se ha posicionado como una fuerza motora de la cultura de la calidad y del control y, por consiguiente, no se ha reconocido su importancia como elemento fundamental para la definición de la cultura organizacional.

La debilidad en los flujos de información de doble vía, que deberían facilitar la retroalimentación, tanto de la gestión institucional como del SGC, incide en el significativo nivel de insatisfacción que se percibe frente a la comunicación interna y a la información sobre el SGC.

Se advierte entonces una clara oportunidad de mejorar los niveles de participación de los servidores públicos en el desarrollo del SGC (desde la comprensión, hasta la toma de conciencia, compromiso, sentido de pertinencia e incidencia) si se fortalecen aspectos como la cultura del autocontrol, el liderazgo y las capacidades de aprendizaje, a través de acciones orientadas a materializar la comunicación desde una perspectiva estratégica integradora y hologramática, cultural, operativa y de crecimiento individual y colectivo.

El compromiso con el desarrollo del SGC será mayor en la medida en que los conceptos y beneficios de este sean mejor comprendidos y que exista una clara intención de utilizarlos para escalar los resultados de la gestión institucional (sentido de pertenencia).

El tema de la calidad se ha renovado otra vez en la discusión permanente sobre la modernización del Estado, ya que es una importante alternativa para "fortalecer la legitimidad de los Gobiernos por medio del rediseño de las instituciones, la mejora continua y el énfasis en un mayor rendimiento de las organizaciones" (Moyado, 2002, p. 9). Es hora tal vez de imprimirle una nueva perspectiva: la necesidad de comunicar eficazmente lo que se quiere de la calidad en la gestión pública, para que los servidores del Estado no solo lo comprendan, tomen conciencia de él y lo sientan propio, sino para que además se comprometan a fondo con su desarrollo y puedan incidir de manera positiva en sus resultados. Así, pues, uno de los mayores retos a corto plazo para las entidades analizadas será sin duda desarrollar mejores metodologías de comunicación interna que favorezcan el desarrollo de los modelos de gestión de la calidad y de cualquier otro que se considere —en el 
largo y complejo camino de la gestión de la calidad y la excelencia que tenga como destino una función pública más eficaz- responsable y comprometida con la construcción de país.

Teniendo en cuenta que el objetivo de este artículo es presentar los resultados sobre el diagnóstico del papel de la comunicación interna y su incidencia en la participación de los servidores públicos en los sistemas de gestión de calidad en entidades del sector gubernamental, una de las primeras recomendaciones que se derivan de esta es precisamente la necesidad de ampliar el estudio a otras entidades públicas, considerando las ramas del poder público (ejecutivo, judicial y jurisdiccional), su alcance territorial (nacional, distrital, gubernamental o municipal) y según su tamaño (en presupuesto y personal) para poder contar con resultados más aproximados a la realidad de la gestión de la calidad en el sector estatal, pues los pocos que existen no permiten llegar a conclusiones claras y contundentes.

La adopción de la cultura de la calidad como modelo de gerencia pública puede llegar a ser más que una moda pasajera, pues como bien lo señala Moyado (2002), "las administraciones públicas experimentan diferentes transformaciones a partir de los cambios que el Estado mismo ha sufrido como consecuencia de un conjunto de reformas que sin duda siguen en curso" (p 1). Por lo tanto, la evolución de la gerencia pública seguirá su devenir de manera simultánea y como respuesta a las nuevas necesidades sociales que las entidades estatales pretenden solventar. En este contexto, los modelos de gestión seguirán probándose, no como experimentos temporales, sino como verdaderos esfuerzos por alinear la administración pública con las tendencias de gestión organizacional. Hoy en día, modelos de gestión pública como el MECI y la gestión de la calidad están en boga en todas las entidades estatales colombianas, con mayor o menor grado de éxito, donde la participación del servidor público ha sido fundamental, y para ello la comprensión de estos modelos resulta importante y significativa.

Esta comprensión del SGC toma como punto de partida la decisión no solo de informar sobre este, sino también de construirlo colectivamente. Para ello, se debe considerar la cultura organizacional y las capacidades de aprendizaje de cada institución, así como la articulación con los componentes estratégicos y las funciones misionales de la entidad. Esta concepción representa una nueva interpretación de los contenidos del enfoque constructivista de la comunicación abordado por Berger y Luckmann (1999), y el trabajo de Formanchuk (2015), pues le imprime una nueva dimensión: poder participar para incidir.

En efecto, la comprensión y el compromiso con el SGC se verán afectados por la capacidad operativa de la entidad para gestionar y materializar los contenidos comunicativos sobre la calidad. No es suficiente con generar flujos de información descendente; es necesario ahondar en prácticas comunicativas más orientadas a la construcción colectiva y participativa de metodologías de gerencia pública.

En este sentido, es necesario conocer y hacer visible el porqué y el para qué de los sistemas de gestión, sus usos y beneficios, pero también sus dificultades y posibles perjuicios. Es conveniente entonces escuchar y escudriñar sobre las diferentes opiniones y posiciones que pueden suscitarse alrededor de la implementación de un SGC. La dialógica mencionada por Morín (citado por Elorriaga et al., 2012) como pilar de la comunicación implica tener en cuenta todas las voces, incluso las disonantes. Esta dialógica también se debe considerar a la hora de estructurar programas de gestión del cambio, que a menudo acompañan la implementación de modelos de gerencia pública, pues los agentes de cambio, es decir, los funcionarios públicos que 
materializan las instituciones públicas, se cuestionan sobre si la implementación de estos modelos mengua su poder de intervención (Hartasánchez, 2008). Aquí la comunicación interna resulta sustancial para mejorar la comprensión del SGC y posibilitar el compromiso y la toma de conciencia, y no se trata solo de pasar del carácter informativo al de consulta, sino más bien de propiciar bases de incidencia y verdadera participación.

Resulta también indispensable abrir el debate sobre el papel que se le ha dado a la comunicación interna en las metodologías y modelos de gerencia adoptados en el sector público colombiano, pues, desde su concepción, el Sisteda, el MECI y el SGC han considerado la comunicación como un elemento esencial para promover la participación en la gestión y la democratización de la información pública. No obstante, en los dos modelos no se trasciende el papel informativo de la comunicación, y queda excluido su papel de facilitador de la incidencia y, por tanto, de la participación.

En la norma técnica ISO 9001:2015 se avanza hacia la concreción de la comunicación interna desde el modelo periodístico de las $5 \mathrm{~W}$ H: qué, quién, cuándo, cómo y a quién. No obstante, es necesario desarrollar una concepción y práctica de la comunicación interna que favorezca la construcción de relaciones sociales, la convivencia y la participación (Berger y Luckmann, 1999), y que contribuya a la construcción del conocimiento (Marchiori, 2011).

La relación íntima entre la comunicación interna, la gerencia estratégica, la gestión del conocimiento y la gestión del cambio no ha sido considerada en su totalidad en la estructura de alto nivel que trae la ISO 9001:2015 y mucho menos en el MECI y la norma de calidad que se aplica en el sector público. Una comunicación de estas características requiere no solo una metodología que complemente lo ya definido por los modelos de gestión de calidad (NTCGP 1000:2009 e ISO 9001:2015) y de control (MECI) adoptados en el sector público, sino que además debe considerar aspectos claves para gobernar y gestionar la comunicación, definir contenidos de acuerdo con los intereses y expectativas de los públicos internos y externos, evaluar impactos y generar sinergias para así articular liderazgos y favorecer la apropiación de estos modelos y su relacionamiento con la gerencia de lo público desde la construcción colectiva.

Al respecto, como se plantea en la Norma para la Evaluación de la Gestión Ética y Socialmente Responsable en las Organizaciones SGE 21:2008 (Forética 2008), es recomendable también considerar la necesidad de identificar y clasificar los diferentes grupos de interés, así como la forma de conocer sus expectativas, a la hora de construir una estrategia y un plan de comunicaciones, y para priorizar las acciones comunicativas que se pueden adelantar en un contexto determinado.

Desde el punto de vista teórico, conceptual y metodológico, se debe avanzar en la construcción de un modelo de comunicación interna para el sector gubernamental, que se complemente con los desarrollos ya existentes para otros temas de gerencia pública como la planeación estratégica, la gestión de riesgos, la administración financiera o el empleo público; pero que también expanda su exploración desde los límites de lo nacional para conocer y confrontar los modelos desarrollados de otros países, desde un enfoque interdisciplinario, que vaya más allá de la administración, la ingeniería industrial o el desarrollo organizacional, y pase por la sociología, la antropología y la lingüística, por ejemplo.

De manera inicial, se propone revisar e incorporar elementos de la construcción colectiva sobre las realidades institucionales (como los que están presentes en la metodología de investigación-acción-participación); 
las nuevas tendencias en medios, contenidos, usos e intercambios comunicativos (como los presentes en la comunicación digital y el endomarketing); la gestión del cambio, en referencia a la importancia de evaluar el impacto que tienen los modelos de gestión en la cultura organizacional (Anderson, 2001) y su articulación con la educación y el aprendizaje (Kotter y Schlesinger, 2008); la gestión del conocimiento, para entender cómo se crea, comprende y se toma conciencia del conocimiento institucional, entre otras tendencias que pueden articularse y complementarse con la comunicación organizacional.

Todo esto como premisa fundamental para estructurar un modelo con énfasis en la comunicación interna, para los SGC y de otros sistemas de gestión, en el ámbito público y privado de la gestión y la gerencia integral.

\section{REFERENCIAS}

Aguilera, J. (2014). Comunicación y calidad: un cambio en la cultura comunicativa. Recuperado de http:// www.degerencia.com/articulo/comunicacion_y_ calidad_un_cambio_en_la_cultura

Aguilera, J. y Orjuela, G. (2008). Estado de la comunicación interna en entidades del Distrito. Recuperado de http://comunicacionparaentidadesdegobierno. blogspot.com/2009/03/estado-de-la-comunicacion-interna-en.html

Anderson, D. (2001). Beyond Change Management. Recuperado de http://gestiondelcambio.com.es/

Berger, P. y Luckmann, T. (1999). La construcción social de la realidad. Buenos Aires: Amorrortu Editores.

Bernal, C. (2010). Metodología de la investigación administración, economía, humanidades y ciencias sociales. Bogotá: Pearson.
Centro Latinoamericano de Aministración para el Desarrollo. (2008). Carta Iberoamericana de la Gestión Pública. Recuperado de http://old.clad.org/ documentos/declaraciones/carta-iberoamericana -de-calidad-en-la-gestion-publica/view

Cilliers, P. (1998). Complexity and Postmodernism. Understanding complex systems. Recuperado de http://14.139.206.50:8080/jspui/bitstream/1/1690 /1/Cilliers, \%20Paul\%20-\%20Complexity\%20 and $\% 20$ Postmodernism.pdf

Cilliers, P. (1998). Complexity and Postmodernism. Understanding complex systems. Recuperado de http://14.139.206.50:8080/jspui/bitstream/1/1690/ 1/Cilliers,\%20Paul\%20-\%20Complexity\%20 and\%20Postmodernism.pdf

Congreso de Colombia. (2014). Decreto 943 de 2014. (21 de mayo de 2014). Por el cual se actualiza el Modelo Estándar de Control Interno (MECI). Diario Oficial. Bogotá, 2014. no. 49158 [en línea]. [Citado el 15 de marzo de 2015]. Recuperado de http://www.alcaldiabogota.gov.co/sisjur/normas/ Norma1.jsp?i=59048.

Contraloría General de la República (2015). Informes de auditoría gubernamental. Recuperado el primero de marzo de 2015, de www.contraloriagen.gov. co/documents/10136/186008126/014_A_MINTRANSPORTE.pdf

De Almeida, M. (2008). Para comprender la complejidad. Multiversidad mundo real Edgar Morín. México, D. F. A.C. Hermosillo.

Elorriaga, K., Lugo, M. y Montero, M. (2012). Nociones acerca de la complejidad y algunas contribuciones al proceso educativo. TELOS, 14(3), 418-424. 
Escobar, J. y Cuervo, Á. (2008). Validez de contenido y juicio de expertos: una aproximación a su utilización. Recuperado de http://www.humanas.unal.edu.co/ psicometria/files/7113/8574/5708/Articulo3_Juicio_de_expertos_27-36.pdf

Forética. (2008). Sistema de gestión ética y socialmente responsable. SGE 21:2008. Recuperado de http:// www.collaboratio.net/mm/File/es/NormaSGE21cast.pdf

Formanchuk, A. (2015). Cómo hacer un plan de comunicación interna: el modelo de las 7 dimensiones. Recuperado de http://formanchuk.com.ar/todosignifica/ comunicacion-interna-7-dimensiones/

Hartasánchez, J. (2008). Gestión del cambio en el sector público. En XIII Congreso Internacional del CLAD sobre la Reforma del Estado y de la Administración Pública. Buenos Aires, Argentina.

Hernández, R., Fernández, C. y Baptista, P. (2014). Metodología de la investigación. México, D. F.: McGraw-Hill.

Icontec. (2009). Sistema de Gestión de la Calidad para la Rama Ejecutiva del Poder Público y otras Entidades Prestadoras de Servicios. Requisitos. NTCGP 1000:2009. Bogotá: El Instituto.

Jackson, P. R. y Welch, M. (2007). Rethinking internal communication: a stakeholder approach. Corporate Communications: An International Journal, 12(2), 177-198. Recuperado de http://www.emeraldinsight.com/doi/full/10.1108/13563280710744847

Kotter, J. P. y Schlesinger, L. A. (2008). Choosing Strategies For Change. Harvard Bussines Review. Recuperado de https://hbr.org/2008/07/choosing -strategies-for-change
Marchiori, M. (2011). Comunicación Interna una visión más amplia en el contexto de las organizaciones. Ciencias de la información. Experiencias del Instituto de Información Científica y Tecnológica. Ciencias de la Información en Revista: Ciencias de la Información, 42(2), p 49-54.

Marín, L. (1997). La formación para la participación y la comunicación en las organizaciones. Reis: Revista española de investigaciones sociológicas, 77(78), 263-282.

Morin, E. (1994). Introducción al Pensamiento Complejo. Recuperado de http://bit.ly/1htogmh

Moyado, F. (2002). Gestión pública y calidad: hacia la mejora continua y el rediseño de las instituciones del sector público. En VII Congreso Internacional del CLAD sobre la Reforma del Estado y de la Administración Pública. Lisboa, Portugal.

Pérez, R. (febrero-agosto, 2012). Comunicación estratégica si claro. Pero ¿qué implica estratégica? Revista Académica de Comunicación y Ciencias Sociales, (2). Recuperado de http://www.tendencias21.net/ estrategar/docs/Metacomunicacion\%202012.pdf

Pérez, R. A. (9 de junio de 2008). Edgar Morín: sobre estrategia. Recuperado de http://www.tendencias21.net/ estrategar/docs/Metacomunicacion\%202012.pdf

Real Academia de la Lengua (2016). Incidencia. Disponible en http://dle.rae.es/srv/search?m=30\&w= incidencia

Rodríguez, T. (2012). La calidad y la mejora en la administración píblica. Madrid: Aenor Ediciones.

Usaid - Casals \& Associates Inc. (2004). Comunicación Pública Estrategias. Modelo de comunicación pública orranizacional e informativa para entidades del Estado, MCPOI - Versión I. Colombia: USAIDCASALS \& ASSOCIATES Inc. 